\title{
Painel amostral para estimativa de áreas agrícolas
}

\author{
Marcos Adami( ${ }^{(1)}$, Mauricio Alves Moreira(1), Bernardo Friedrich Theodor Rudorff(1), Corina da Costa Freitas ${ }^{(1)}$, \\ Rogério Teixeira de Faria ${ }^{(2)}$ e Flavio Deppe ${ }^{(3)}$
}

\begin{abstract}
(1)Instituto Nacional de Pesquisas Espaciais, Av. dos Astronautas, no 1.758, Jardim da Granja, Caixa Postal 515, CEP 12201 -970 São José dos Campos, SP. E-mail: adami@Itid.inpe.br, mauricio@Itid.inpe.br, bernardo@Itid.inpe.br, corina@dpi.inpe.br (2)Instituto Agronômico do Paraná, Rod. Celso Garcia Cid, Km 375, Três Marcos, Caixa Postal 481, CEP 86047-902 Londrina, PR. E-mail: rtfaria@iapar.br (3)Instituto Tecnológico Simepar, Centro Politécnico da Universidade Federal do Paraná, Jardim das Américas, Caixa Postal 19.100 CEP 81531-990 Curitiba, PR. E-mail: deppe@simepar.br
\end{abstract}

Resumo - O objetivo deste trabalho foi desenvolver metodologia que integre sensoriamento remoto e sistema de informações geográficas (SIG) para criar um painel amostral e alocar segmentos regulares por meio de um sistema de amostragem aleatória estratificada. Diferentes estratos foram obtidos por meio da classificação de imagens de satélite. Em cada estrato foi realizado sorteio para a seleção dos segmentos amostrais, os quais foram visitados para delimitar as diversas culturas e estimar a área. Na estimativa da área plantada por cultura, para toda a região, utilizou-se o estimador por expansão direta por meio de um procedimento semi-automático. A metodologia reduziu tanto a subjetividade do percentual da área agrícola estimada no estrato, quanto o tempo de construção do painel amostral.

Termos para indexação: estatísticas agrícolas, sensoriamento remoto, SIG.

\section{Sampling frame for crop area estimation}

\begin{abstract}
The objective of this work was to develop a methodology in order to use remote sensing and Geographic Information System (GIS) to construct a sampling frame and to allocate regular segments in a region through a random stratified sampling system. The different strata were obtained through satellite image classification. In each stratum a drawing was carried out to select sample segments, which were field visited to identify the crop type and to estimate their area. In the area estimation for each crop, for the entire region, the direct expansion estimator through a semi-automated procedure was used. The methodology reduced both the subjectivity of the percentage of agricultural area estimation in the strata and the time to construct the sampling frame.
\end{abstract}

Index terms: agricultural statistics, remote sensing, GIS.

\section{Introdução}

O emprego de técnicas de amostragem para gerar estimativas de populações é amplamente utilizado, principalmente em virtude dos fatores tempo e economia. O Brasil precisa de um sistema de previsão de safras que ofereça dados confiáveis em tempo hábil e que permita o planejamento de ações (Adami et al., 2005; Rizzi et al., 2006).

Exemplos de métodos de estimativas de safras que utilizam sensoriamento remoto e Sistema de Informação Geográfica (SIG), em diversos países, foram relatados pela FAO (1998) e por Gallego (2004). No Brasil, a primeira metodologia desenvolvida para estimar áreas de culturas agrícolas por meio de amostragem probabilística de áreas envolvendo imagens de satélites foi o projeto pesquisa de previsão e acompanhamento de safras (PREVS) (FAO, 1998), com as características de desenho probabilístico; construção de painel de amostragem de áreas; seleção de amostra probabilística; aplicação de estimadores por expansão direta; e de painéis múltiplos (Mueller et al., 1988). Apesar da dimensão do projeto quanto à área abrangida, as imagens de satélite foram utilizadas somente na fase de estratificação da área e, mesmo assim, por um processo de interpretação visual que consumiu horas de trabalho. Ademais, o material levado a campo para orientar os coletadores de dados sobre o uso e ocupação do solo, nos segmentos da amostra, incluía limites dos segmentos sobrepostos às fotografias aéreas métricas.

Em virtude do custo de aquisição e restituição aerofotogramétrica, as fotografias usadas não eram atualizadas, e, freqüentemente, informações sobre uso 
do solo e limites dos segmentos no campo não correspondiam aos representados nas fotografias. Esses entraves influenciaram a desativação do projeto em 2000, e, assim, as estimativas oficiais brasileiras de área cultivada continuaram sendo realizadas somente pelo sistema subjetivo.

O sistema subjetivo de previsão de área cultivada baseia-se na opinião de agentes técnicos e econômicos ligados ao agronegócio. Esse sistema utiliza informações referentes à quantidade de insumos comercializados, crédito agrícola, especulações sobre as condições meteorológicas, tendência de mercado e histórico da região, entre outras, que não permitem análise quantitativa dos erros envolvidos, além de serem passíveis de manipulação (Pino, 2001).

A evolução da informática (Pino, 1999) e a disponibilidade maior de imagens de satélite de observação da Terra tornaram possíveis o acompanhamento e o monitoramento das áreas agrícolas de forma mais eficaz. A integração desses dois elementos aos modelos estatísticos permite a obtenção de informações de forma rápida e objetiva a um custo reduzido. Essa afirmativa exprime a necessidade de implementação de um modelo robusto para ser aplicado em áreas agrícolas extensas, como é o caso do Brasil, em que toda metodologia visual de construção do painel amostral seja implementada em um SIG, tornando os procedimentos mais eficientes e menos subjetivos, sobretudo na estratificação do uso do solo. Associado a isso, ocorreu a liberação do sinal de Global Positioning System (GPS) sem ruído, o que tornou os receptores de navegação mais precisos e possibilitou alterar o formato dos segmentos.

Este trabalho teve como objetivo desenvolver metodologia que integre sensoriamento remoto e sistema de informações geográficas (SIG) para criar um painel amostral e alocar segmentos regulares por meio de um sistema de amostragem aleatória estratificada.

\section{Material e Métodos}

A região do estudo localiza-se ao norte do Estado do Paraná, e abrange 23 municípios pertencentes ao núcleo regional da Secretaria da Agricultura e do Abastecimento do Estado do Paraná (Seab), com área de aproximadamente $7.500 \mathrm{~km}^{2}$, compreendida entre as coordenadas $22^{\circ} 45^{\prime} 35^{\prime \prime}$ a $24^{\circ} 03^{\prime} 35^{\prime \prime} \mathrm{S}$ e $50^{\circ} 08^{\prime} 00^{\prime \prime}$ a $51^{\circ} 12^{\prime} 53^{\prime \prime W}$. Nessa área, a atividade agrícola é predominante, e os municípios ao norte possuem relevo mais plano, pouco suscetível à erosão e mecanizáveis em grande parte da área. Ao sul o relevo é acidentado e a mecanização é empregada de forma restrita (Brasil, 1981).

O banco de dados geográficos foi criado pelo software SPRING (Câmara et al., 1996), no qual foram inseridas: imagens do satélite Landsat-7 sensor ETM $^{+}$(Enhanced Thematic Mapper plus), correspondentes ao ponto 76 das órbitas 221 e 222, tanto para 2001 (para estratificação) quanto para 2002 (para impressão do material de campo); elementos cartográficos; informações cadastrais e estatísticas e dados coletados no campo.

$\mathrm{Na}$ etapa de estratificação, utilizaram-se os limites dos estratos adotados pela PREVS, para o Estado do Paraná de acordo com Mueller et al. (1988) e FAO (1998). Foram definidos seis estratos com as seguintes características: estrato A, $80 \%$ ou mais de área cultivada; estrato B, 50 a 79\% de área cultivada; estrato C, 15 a 49\% de área cultivada, com predominância de pastagens; estrato $\mathrm{D}$, menos de $15 \%$ de área cultivada; estrato E, 15 a 49\% de área cultivada, sem a predominância de pastagens; e estrato $\mathrm{F}$, áreas não agrícolas. $\mathrm{O}$ estrato $\mathrm{F}$ não foi amostrado.

Depois de definir os estratos, a etapa seguinte foi a classificação não-supervisionada das imagens por meio do classificador ISOSEG (Inpe, 2006), para obtenção da distribuição espacial dos diferentes usos do solo. As imagens de 2001 utilizadas na construção do painel amostral se referem ao período da entressafra, quando a maioria das terras utilizadas na agricultura se encontrava com a aparência de solo exposto, nas imagens, e, portanto, facilitou o procedimento de classificação. Cada classe de uso e ocupação do solo foi separada em diferentes planos de informações (PI), realizando-se a interpretação visual, na tela do computador, por meio da ferramenta de edição matricial, segundo recomendações descritas por Crepani et al. (2002). O mapa resultante foi composto por cinco classes temáticas: área agrícola; corpos d'água; mata; pastagem; e área urbana.

A partir do mapa temático obtido na fase de classificação, estratificou-se a área segundo as características definidas para a PREVS, de acordo com Mueller et al. (1988). A fim de obter a porcentagem de área das diferentes classes, o mapa temático foi desdobrado em cinco imagens binárias, com valor 100 para a classe desejada e zero para as demais classes. Em seguida, foi utilizado o segmentador por crescimento de regiões sobre as imagens binárias, cujos 
parâmetros de entrada foram: bandas, selecionar todos os PI's provenientes da classificação, transformados em imagens; método, crescimento de regiões; limiar de similaridade, 1, para individualizar as menores mudanças; e limiar de área, em função do tamanho do pixel e tamanho mínimo do estrato. O cálculo foi realizado de modo que áreas menores de $20 \mathrm{~km}^{2}$ não fossem individualizadas. Logo, o tamanho do polígono (P) resultante da segmentação foi determinado pela equação:

$\mathrm{P}=\frac{\text { Apol }}{\text { Apix }}$ ou $\mathrm{P}=\frac{2.10^{7} \mathrm{~m}^{2}}{900 \mathrm{~m}^{2}}$

em que, Apol é a menor área a ser individualizada e Apix é a área do pixel das imagens resultantes da classificação.

A fim de garantir a equivalência entre os limites dos estratos e o limite dos segmentos (Gallego, 1995; Pradhan, 2001), foi obtido um mosaico, contendo os limites vetoriais definidos pelo segmentador para uma classe temática com resolução igual à da amostra $\left(1 \mathrm{~km}^{2}\right)$. Depois desse procedimento, cada polígono foi associado a uma classe. Posteriormente, foi feito o cálculo do porcentual de área utilizada para cada classe em cada polígono, por meio do operador de média zonal.

Na seqüência, a área de estudo foi dividida em segmentos regulares de $1 \mathrm{~km}^{2}$, e cada polígono recebeu um identificador. Para separar a grade regular de segmentos por estrato, foi realizada consulta espacial com a operação topológica "dentro", a fim de selecionar todos os elementos da grade contidos dentro do estrato e que não tocavam a linha do polígono do estrato. Adicionouse à consulta anterior, a operação topológica "coberto por", somando-se todas as unidades amostrais que tocavam a linha do polígono por dentro (Figura 1).

Para determinar o tamanho da amostra, foi empregada a fórmula proposta por Thompson (2002), segundo a Equação 2:

$\mathrm{n}=\frac{\left(\mathrm{Z}_{\alpha / 2}\right)^{2} \mathrm{pq}}{\mathrm{E}^{2}}$

em que, né o número total de segmentos que é amostrado para estimar a área com determinado porcentual de confiança $(\alpha / 2)$ e um erro de amostragem (E); p é o porcentual de área dentro do segmento ocupado com agricultura; q é a área utilizada com outras ocupações do solo, dentro do segmento, representado por (1-p), e $\mathrm{Z}$ é o valor crítico tabelado da função normal-padrão, quanto ao intervalo de confiança $(\alpha / 2)$.

O número de segmento por estrato (nh) foi determinado pela Equação 3:

$$
\mathrm{nh}=\frac{\mathrm{Nh} \cdot \mathrm{Sh} / \sqrt{\mathrm{Ch}}}{\sum^{\mathrm{Nh}} \cdot \mathrm{Sh} / \sqrt{\mathrm{Ch}}} \cdot \mathrm{n}
$$

em que, Nh é o número total de elementos do estrato; Sh é o desvio-padrão do estrato; Ch é o custo da amostragem, calculado de acordo com o porcentual de uso do solo pela cultura de interesse, definido por $\mathrm{Ch}=1 / \mathrm{ph}$, em que ph é o porcentual do segmento ocupado pela classe, obtido pelo operador de média zonal; n é o número total de segmentos que deve ser amostrado na área.

Em seguida, realizou-se o sorteio aleatório dos segmentos em cada estrato. Os segmentos sorteados foram impressos sobre as imagens de 2002 (data próxima ao trabalho de campo), de modo a facilitar sua localização no campo, bem como determinar os limites dos talhões contidos no interior de cada segmento (Adami, 2003).

O trabalho de campo para coleta de dados, nos segmentos da amostra, teve início em 29/10/2002 e terminou em 10/12/2002. A localização das amostras foi realizada com auxílio de um receptor GPS de navegação. Ao se localizar o segmento, o material impresso serviu de referência para delimitar os talhões com as culturas. No caso dos limites dos talhões não serem visíveis na imagem, foi realizado o caminhamento em torno do talhão com o receptor GPS.

Os dados coletados a campo foram utilizados no modelo de expansão direta para estimar a área dos alvos de ocupação do solo, conforme a equação:

$\hat{\mathrm{Z}}_{\mathrm{c}}=\sum_{\mathrm{i}=1}^{\mathrm{m}} \mathrm{e}_{\mathrm{i}} \sum_{\mathrm{k}=1}^{\mathrm{n}_{\mathrm{i}}} \mathrm{z}_{\mathrm{c}, \mathrm{ik}}$

em que, $\hat{Z}$ é o estimador da área; c é o índice para representar a cultura de interesse; i é o índice para representar o estrato de uso do solo com $\mathrm{i}=1, \ldots, \mathrm{m}$; $\mathrm{k}$ é o índice para representar o segmento amostrado com $\mathrm{k}=1, \ldots, \mathrm{n}_{\mathrm{i}} ; \mathrm{m}$ é o número de estratos; $\mathrm{n}_{\mathrm{i}}$ é o número de segmentos amostrados no i-ésimo estrato de uso do solo; $\mathrm{e}_{\mathrm{i}}$ é o fator de expansão ou inverso da probabilidade, que determinado segmento tem de estar na amostra escolhida, no i-ésimo estrato de uso do solo, sendo definido por $\mathrm{e}_{\mathrm{i}}=\left(\mathrm{n}_{\mathrm{i}} / \mathrm{N}_{\mathrm{i}}\right)^{-1}$, em que $\mathrm{N}_{\mathrm{i}}$ é o número total de segmentos no i-ésimo estrato de uso do solo; e $\mathrm{z}_{\mathrm{c}, \mathrm{ik}}$ é a área da cultura c, no k-ésimo segmento do i-ésimo estrato de uso do solo.

A variância estimada de $\hat{\mathbf{Z}}_{c}$ é definida pela equação:

$$
\mathrm{v}\left(\hat{\mathrm{Z}}_{\mathrm{c}}\right)=\sum_{\mathrm{i}=1}^{\mathrm{m}} \mathrm{N}_{\mathrm{i}}\left(\mathrm{N}_{\mathrm{i}}-\mathrm{n}_{\mathrm{i}}\right) \frac{\mathrm{s}_{\mathrm{c}, \mathrm{i}}}{\mathrm{n}_{\mathrm{i}}}
$$


em que, $\mathrm{S}_{\mathrm{c}, \mathrm{p}}^{2}$ é a variância estimada da cultura c no estrato i, definida pela equação:

$S_{c, i}^{2}=\frac{\sum_{k=1}^{n_{i}}\left(z_{c, i k}-\bar{z}_{c, i}\right)^{2}}{\left(n_{i}-1\right)}$

e $\bar{Z}_{\mathrm{c}, \mathrm{i}}$ é a área média da cultura c no i-ésimo estrato de uso do solo, definida pela equação:

$\bar{z}_{\mathrm{c}, \mathrm{i}}=\frac{\sum_{\mathrm{k}=1}^{\mathrm{n}_{\mathrm{i}}} \mathrm{z}_{\mathrm{c}, \mathrm{i}}}{\mathrm{n}_{\mathrm{i}}}$

O coeficiente de variação $(\mathrm{CV})$ é calculado pela equação:

$\mathrm{CV}(\%)=\frac{\sqrt{\frac{\sum_{\mathrm{k}-1}^{n}\left(\mathrm{Z}_{\mathrm{c}, \mathrm{k}}-\bar{Z}_{\mathrm{ci}}\right)^{2}}{\left(\mathrm{n}_{\mathrm{i}}-1\right)}}}{\hat{\mathrm{Z}}_{\mathrm{c}}} * 100$

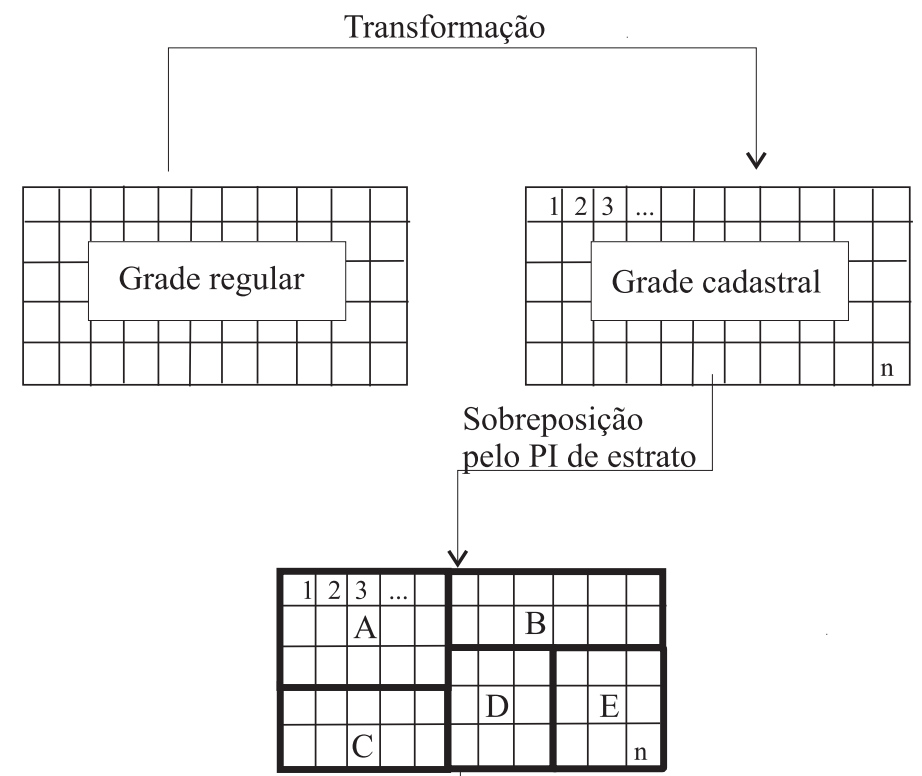

Consulta espacial

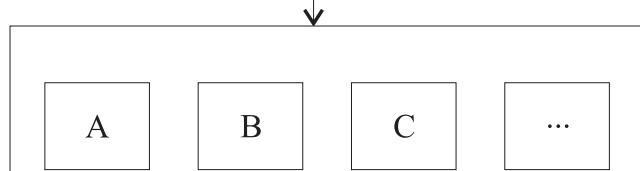

PI contendo cada elemento por estrato

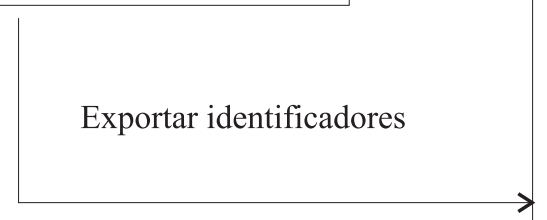

$>$

dentificadores

estrato “..."

\section{Resultados e Discussão}

A aplicação da metodologia para estratificar a região de estudo por meio do algoritmo de segmentação por crescimento de regiões resultou na detecção de diversas regiões homogêneas. Entretanto, ao sobrepor o PI de polígonos com o PI das amostras, as linhas das regiões homogêneas, delimitadas pelo segmentador, não coincidiram com os limites da grade gerada com $1 \mathrm{~km}^{2}$ de resolução, que corresponde ao tamanho da unidade amostral (Figura $2 \mathrm{~A}$ ).

Com o estrato obtido pela segmentação, sem o ajuste dos polígonos aos limites dos segmentos, o estabelecimento de um sistema de amostragem ale-

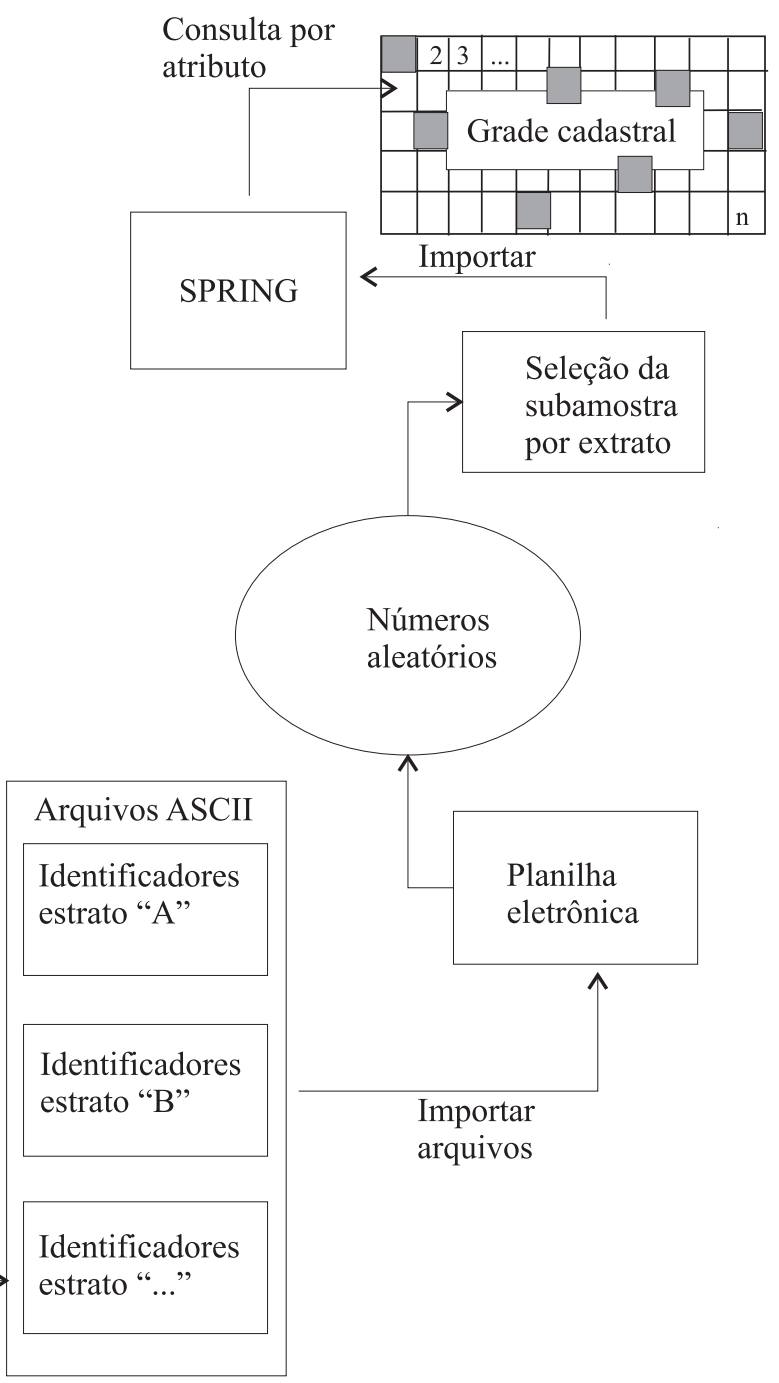

Figura 1. Procedimentos para a criação e sorteio dos segmentos. 
atória estratificada se tornaria difícil de ser implementado, pois o segmento pode pertencer a vários estratos ao mesmo tempo (Gallego, 1995; Pradhan, 2001).

$\mathrm{O}$ ajuste vetorial entre os limites dos estratos e os segmentos permite que cada segmento pertença
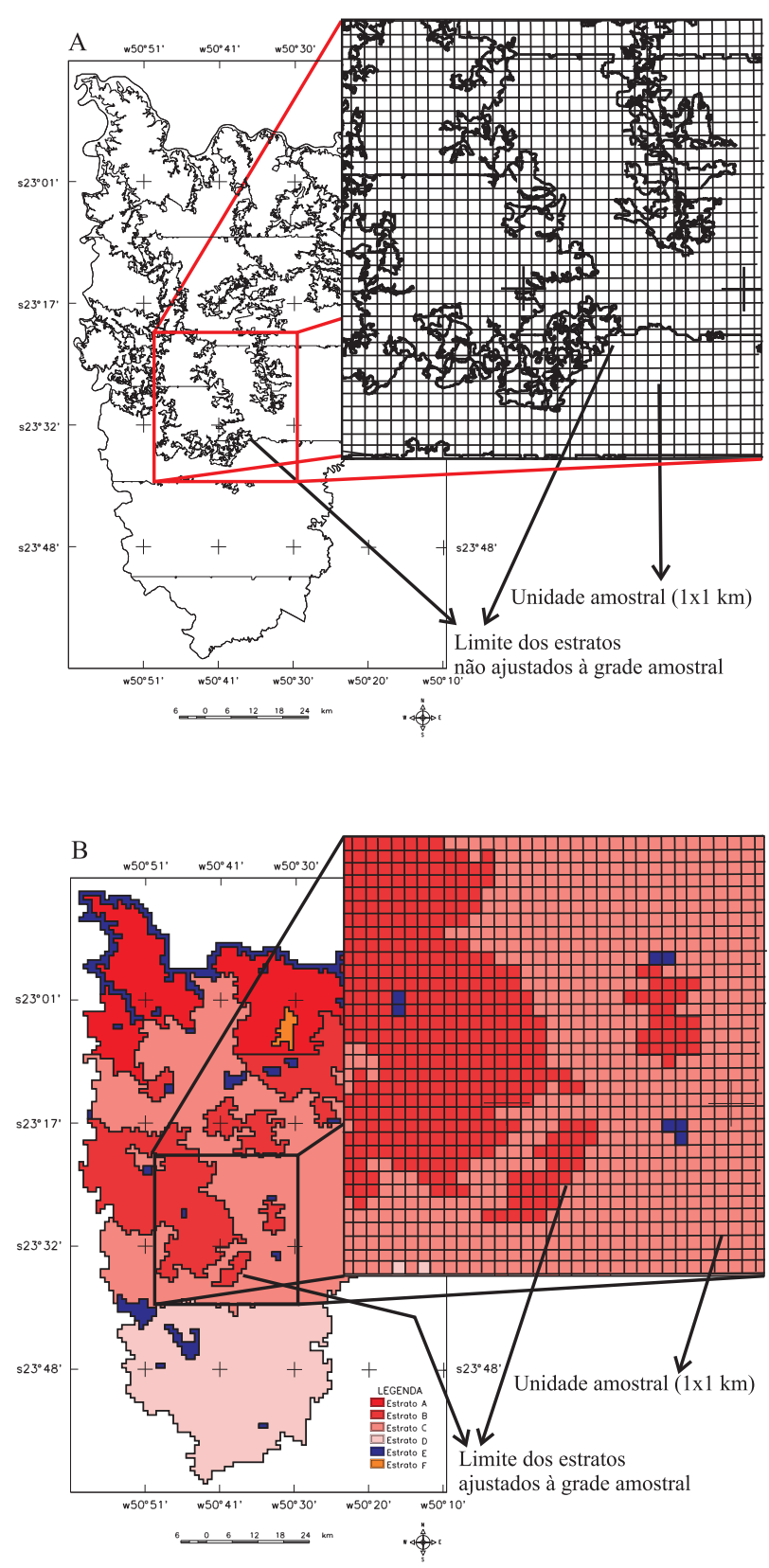

Figura 2. Polígonos obtidos pela segmentação (A) e ajustados aos limites dos segmentos (B), sobrepostos pelos limites das unidades amostrais. a apenas um estrato (Figura 2 B). Apesar disso, este procedimento altera as bordas do estrato, pois é dado de acordo com a dimensão da amostra. No caso do SPRING, o pixel da borda do segmento pertence à mesma classe do centróide do pixel sobreposto com ele. Por meio desse critério, esperase que a mudança de área e de classe seja estabelecida de acordo com a resolução espacial e que, no limite, as alterações de borda tendam à média, não alterando drasticamente os valores originais do polígono. A integração de dados cadastrais e de sensoriamento remoto em um SIG é complexa, devido às diferenças nos processos de aquisição e da própria estrutura dos dados (Pradhan, 2001); entretanto, com a integração matriz e vetor utilizada na metodologia foi possível simplificar o procedimento.

Na PREVS, a estratificação foi estabelecida com base na interpretação visual, ao passo que, neste trabalho, foi realizada de forma semiautomática, reduzindo a subjetividade do procedimento. Além disso, a mão-de-obra empregada na construção do painel amostral foi de 21 dias-homem, enquanto na PREVS do Paraná, apenas para obter as unidades primárias de amostragem, foram empregados 72 dias-homem em área equivalente (FAO, 1998).

Por meio da metodologia utilizada, foi possível estratificar a área de estudo em seis estratos de uso do solo, e o estrato F, de áreas não-agrícolas, foi excluído da amostragem. Tanto o resultado da estratificação quanto a localização dos segmentos sorteados podem ser visualizadas na Figura 3.

Ao estratificar a área de estudo, observou-se a relação entre o uso do solo e a localização. Ao norte da região, há alto índice de utilização do solo para a agricultura (estratos A e B), enquanto ao sul e sudeste da região, há um alto índice de utilização do solo para a pecuária (estratos C e D) (Figura 3). Esse resultado está de acordo com o mapeamento das limitações de uso do solo do Estado do Paraná quanto à suscetibilidade à erosão (Brasil, 1981).

Por meio da informação da média de área para agricultura e da Equação 2, foi calculado o número total de segmentos. A subdivisão desses segmentos, 


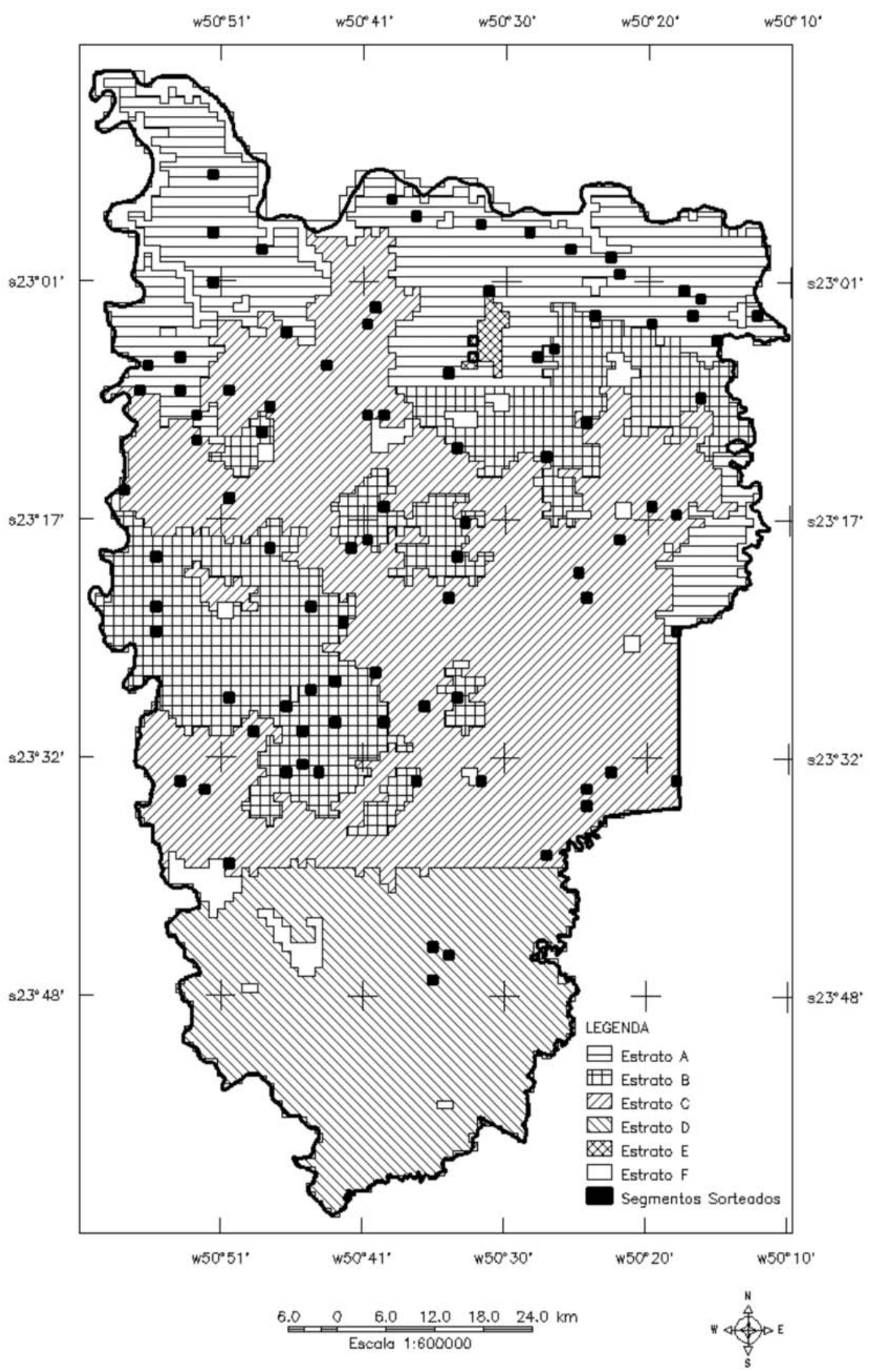

Figura 3. Estratos de uso do solo na região de estudo e localização dos segmentos amostrados. 
de cada uma das subamostras, foi calculada por meio da Equação 3 (Tabela 1).

A área amostrada correspondeu a 1,23\% da área da região. Porcentuais semelhantes foram utilizados em estudos realizados por Hill \& Mégier (1988), no Sul da França, e por Deppe (1996) no Sul do Brasil. No caso da PREVS, o total amostrado para o Estado do Paraná correspondeu a $0,5 \%$ (FAO, 1998).

O resultado das estimativas foi obtido três dias depois do término da coleta de dados de campo, denotando a agilidade de obtenção de informações com o presente método. Além disso, as estimativas geradas estão associadas ao erro (CV), o que permite análise estatística e as torna objetivas.

A área estimada com a cultura da soja corresponde a $33 \%$ da área total estimada (Tabela 2). As áreas estimadas para culturas de milho e de café foram pouco expressivas, quando comparadas com a cultura de soja. Os menores CV para soja e pastagem indicam que as estimativas são mais precisas para as ocupações que possuem maior área.

Tabela 1. Número total de segmentos, tamanho das subamostras e porcentual da área amostrada, por estrato.

\begin{tabular}{cccc}
\hline Estrato & $\begin{array}{c}\text { Número total } \\
\text { de segmentos (a) }\end{array}$ & $\begin{array}{c}\text { Número de segmentos } \\
\text { amostrados (b) }\end{array}$ & $\begin{array}{c}\text { Porcentagem da } \\
\text { área }\end{array}$ \\
\hline $\mathrm{A}$ & 1.416 & 25 & 1,77 \\
$\mathrm{~B}$ & 1.432 & 28 & 1,95 \\
$\mathrm{C}$ & 2.919 & 31 & 1,06 \\
$\mathrm{D}$ & 1.404 & 3 & 0,21 \\
$\mathrm{E}$ & 28 & 2 & 7,21 \\
\hline Total & 7.564 & 89 & 1,23 \\
\hline
\end{tabular}

Coeficientes de variação relativamente altos foram observados em culturas de café e de milho, resultado aceitável devido à baixa freqüência de segmentos amostrados e da alta variabilidade na taxa de ocupação dessas culturas, nos segmentos amostrados, causando, também, elevação na variância da estimativa. Fato relevante que demonstra levar em consideração a taxa de ocupação e as formas de cultivo das culturas no processo de estratificação. Altos valores de CV também foram observados para usos do solo em que a área não foi expressiva.

Os CV apresentados na Tabela 2 são semelhantes aos relatados por Mueller et al. (1988), Hill \& Mégier (1988), Gonzáles-Alonso et al. (1991), Gallego (1995), Tsiligirides (1998) e Pradhan (2001). Essa Tabela mostra ainda a relação inversa entre o porcentual de área ocupada pela cultura e o CV, além de fornecer parâmetro objetivo de avaliação da estimativa.

Ao comparar a estimativa de área obtida nesta pesquisa com a estimativa subjetiva gerada pela Seab/ Deral (Paraná, 2006), ambas apresentadas na Tabela 2, observa-se que a maior diferença entre as estimativas foi quanto à cultura do milho, cuja área estimada pela Seab/Deral (Paraná, 2006) foi 57,16\% maior do que a estimada por esta pesquisa. Essa diferença se deve a ocorrência de um veranico, fazendo com que os agricultores que tinham a intenção de plantar milho e ainda não o tinham feito, optassem por plantar soja. Quanto às demais culturas, as diferenças foram bem menores.

Tabela 2. Área estimada pela amostragem $\left(\mathrm{km}^{2}\right)$, variância $\left(\mathrm{km}^{4}\right)$ e coeficiente de variação $(\mathrm{CV}, \%)$ para as variáveis da pesquisa, e área estimada pela Seab $\left(\mathrm{km}^{2}\right)$ e diferença $(\%)$ entre as duas áreas estimadas para as culturas agrícolas da região estudada.

\begin{tabular}{|c|c|c|c|c|c|}
\hline Cultura & $\begin{array}{c}\text { Área estimada pela } \\
\text { amostragem (a) }\end{array}$ & Variância & $\mathrm{CV}$ & $\begin{array}{l}\text { Área estimada } \\
\text { pela Seab (b) }\end{array}$ & $\begin{array}{c}\text { Diferença } \\
100[(b-a) / a]\end{array}$ \\
\hline Café & 159,39 & $1.528,89$ & 24,53 & 152,15 & $-4,54$ \\
\hline Milho & 229,06 & $3.561,24$ & 26,05 & 360,00 & 57,16 \\
\hline Soja & $2.402,61$ & $25.093,51$ & 6,59 & $2.540,00$ & 5,72 \\
\hline Pastagem & $3.285,84$ & $36.711,15$ & 5,83 & - & - \\
\hline Mata & 678,44 & $19.320,23$ & 20,49 & - & - \\
\hline Outros usos agrícolas & 358,42 & $4.932,59$ & 19,59 & 432,58 & 20,69 \\
\hline Não-agropecuário & 85,24 & $1.357,95$ & 43,23 & - & - \\
\hline Total & $7.199,00$ & - & & - & - \\
\hline
\end{tabular}

${ }^{(1)}$ Fonte: Paraná (2006); a Seab/Deral não realiza estimativas de área para pastagem, mata e não-agropecuário. 


\section{Conclusões}

1. A metodologia é adequada para realizar a amostragem e estimar de forma objetiva a área de culturas agrícolas.

2. A metodologia desenvolvida integra sensoriamento remoto e SIG para a construção de um painel amostral de forma semi-automática.

3. É possível estimar o porcentual da área agrícola, por estrato, e ajustar os limites dos estratos aos limites dos segmentos, de forma automatizada.

\section{Referências}

ADAMI, M. Estimativa de áreas agrícolas por meio de técnica de sensoriamento remoto, geoprocessamento e amostragem. 2003. 183p. Dissertação (Mestrado) - Instituto Nacional de Pesquisas Espaciais, São José dos Campos.

ADAMI, M.; MOREIRA, M.A.; RUDORFF, B.F.T.; FREITAS, C.C.; FARIA, R.T. Expansão direta na estimativa de culturas agrícolas por meio de segmentos regulares. Revista Brasileira de Cartografia, v.57, p.22-27, 2005.

BRASIL. Ministério da Agricultura. Paraná (PR): mapa das limitações dos usos dos solos do Estado do Paraná por suscetibilidade à erosão. Brasília, 1981. 1 mapa. Escala 1:600.000.

CÂMARA, G.; SOUZA, R.C.M.; FREITAS, U.M.; GARRIDO, J.C.P. SPRING: integrating remote sensing and GIS with objectoriented data modelling. Computers and Graphics, v.15, p.13-22, 1996.

CREPANI, E.; DUARTE, V.; SHIMABUKURO, Y.E.; FIDALGO, E.C.C. Sensoriamento remoto e geoprocessamento no mapeamento regional da cobertura e uso atual da terra. Geografia, v.27, p.119135, 2002.

DEPPE, F. Forest area estimation using sample surveys and Landsat MSS and TM data. Photogrammetric Engineering and Remote Sensing, v.64, p.13-22, 1996.

FAO. Multiple frame agricultural surveys: agricultural survey programmes based on area frame or dual frame (area and list) sample designs. Roma, 1998. v.2, 242p.
GALLEGO, F.J. Remote sensing and land cover area estimation. International Journal of Remote Sensing, v.25, p.3019-3047, 2004.

GALLEGO, F.J. Sampling frames of square segments. Luxembourg: Joint Research Centre, 1995. 72p.

GONZÁLES-ALONSO, F.; SORIA, S.L.; GOZALO, J.M.C. Comparing two methodologies for crop area estimation in Spain using Landsat TM images and ground-gathered data. Remote Sensing of Environment, v.35, p.29-35, 1991.

HILL, J.; MÉGIER, J. Regional land cover and agricultural area statistics and mapping in the Département Ardèche, France, by use of Thematic Mapper data. International Journal of Remote Sensing, v.9, p.1573-1595, 1988.

INPE (São José dos Campos, SP). Sistema de Processamento de Informações Georeferenciadas - SPRING 3.5.1: tutoriais. Disponível em: <http://www.dpi.inpe.br/spring/portugues/ index.html>. Acesso em: 3 out. 2006.

MUELlER, C.C.; SILVA, G.; VILlalobOS, A.G. Pesquisa agropecuária do Paraná - safra 1986/87 (Programa de Aperfeiçoamento das Estatísticas Agropecuárias). Revista Brasileira de Estatística, v.49, p.55-84, 1988.

PARANÁ. Secretaria de Estado da Agricultura e do Abastecimento. Departamento de Economia Rural. Produção agrícola: comparativo de área e produção. Disponível em: <http://www.pr.gov.br/seab>. Acesso em: 23 jul. 2006.

PINO, F.A. Estatísticas agrícolas para o século XXI. Agricultura em São Paulo, v.46, p.71-105, 1999.

PINO, F.A. Estimação subjetiva de safras agrícolas. Informações Econômicas, v.31, p.55-58, 2001.

PRADHAN, S. Crop area estimation using GIS, remote sensing and area frame sampling. International Journal of Applied Earth Observation and Geoinformation, v.3, p.86-92, 2001.

RIZZI, R.; RUDORFF, B.F.T.; ADAMI, M. Estimativa da área de soja no Estado do Rio Grande do Sul por um método de amostragem. Ciência Rural, v.36, p.30-35, 2006.

THOMPSON, S.K. Sampling. $2^{\text {nd }}$ ed. New York: Wiley, 2002. $343 p$.

TSILIGIRIDES, T.A. Remote sensing as a tool for agricultural statistics: a case study of area frame sampling methodology in Hellas. Computers and Electronics in Agriculture, v.20, p.45-77, 1998.

Recebido em 12 de agosto de 2005 e aprovado em 30 de agosto de 2006 